\title{
Multi-Expansion Modal Reduction: A pragmatic semi-a-priori model order reduction approach for nonlinear structural dynamics
}

\author{
${\text { Frank Naets*1,2 | Daniel De Gregoriis }{ }^{1} \text { | Wim Desmet }}^{1,2}$
}

${ }^{1}$ Department of Mechanical Engineering, KU Leuven, Belgium

${ }^{2}$ Member of DMMS Lab, Flanders Make

\section{Correspondence}

*Frank Naets, Celestijnenlaan 300, 3001

Heverlee, Belgium. Email:

frank.naets@kuleuven.be

\begin{abstract}
Over the past decades, nonlinear finite-element simulation has become a popular tool for a broad range of structural dynamics analyses. However, for many applications the direct application of these models is prohibitively expensive. In order to alleviate this issue, a novel model order reduction scheme is proposed in this work which enables an accelerated model evaluation. The proposed Multi-Expansion Modal (MEM) reduction exploits a set of modal bases evaluated for an number of representative statically deformed configurations, together with these static deformation patterns, to obtain a reduced order basis (ROB). This ROB is combined with an a-priori element sampling to obtain a hyper-reduced model based on the energy-conserving sampling and weighting approach. The proposed element sampling scheme is based on a non-negative $L_{1}$ optimization of the element weights where an equality between the projected and hyper-reduced linearized stiffness matrix or static deformation forces is added. The MEM approach is validated numerically on an academic cantilever beam model and is shown to provide good accuracy and consistent convergence properties.
\end{abstract}

\section{KEYWORDS:}

Nonlinear, Model order reduction, structural dynamics

\section{1 | INTRODUCTION}

As the possibilities of numerical simulation approaches increases, users demand ever increasing accuracy from their models. In many research and industrial mechanical engineering areas, this implies a push to a wider adoption of large nonlinear finite element models. However, for most applications, the use these nonlinear finite elements (NL-FE) models in dynamic timedomain simulations is too large for a wide adoption in engineering practice. Model order reduction (MOR) techniques offer the potential to dramatically speed up these simulations, beyond what can typically be obtained through faster (parallel) hardware and dedicated parallel algorithms.

Model order reduction originates from the study of large scale linear systems, and a wide range of methods have been developed for structural dynamics applications ${ }^{1}$. However, these methods are known for not being suitable to handle nonlinear structural applications, due to specific issues like locking phenomena ${ }^{2}$. Linear MOR only consists of a single stage, being the setup of a low rank reduced order basis (ROB) which allows to approximate the response of the full order model (FOM) and which can be used to project the mass and stiffness matrix such that a low cost model is obtained.

In order to resolve the inadequacy of linear MOR techniques for nonlinear applications, nonlinear model order reduction methods have been developed. For nonlinear model order reduction, typically a two step approach needs to be adopted: 
- Reduction through projection: A low rank reduced order basis needs to be constructed which allows to approximate the full order state of the nonlinear model. This limits the number of degrees-of-freedom (DOFs) for which the model needs to be solved and can reduces the simulation cost.

- Hyper-reduction: In nonlinear (structural) models, a considerable amount of computational time is spent on evaluating the contributions of all nonlinear terms in a nonlinear finite element model. Hyper-reduction allows to approximate the nonlinear internal forces projected on the ROB. Typically this is achieved by selecting the dominant nonlinear contributions in some sense and only evaluating these during the simulation.

The most popular approaches in nonlinear model order reduction are based on a ROB setup from a proper orthogonal decomposition (POD) ${ }^{3.4}$. This ROB is then combined with a dominant nonlinear contribution selection approach. In the discrete empirical interpolation method (DEIM), single force contributions are selected ${ }^{5,6.3}$. However this approach has been shown to often lead to unstable models for elastodynamics as no structure of the FOM is preserved. In order to resolve this issue, the energy conserving sampling and weighting (ECSW) selects dominant elements and performs a nonnegative weighting of these elemental contributions ${ }^{4.7}$. For mechanical applications, this final approach has the main advantage that the Lagrangian structure, and hence the stability, of the FOM is preserved in the reduced order model (ROM). Even though these are very powerful methods which have shown important speed-ups for a range of applications, they require a (set of) reference dynamic simulation(s) of the full order model in order to identify the ROB and dominant nonlinear contributions. For many applications, this limits the applicability in practice as the cost (long throughput time) of the reference simulation(s) is often infeasible.

In order to circumvent this issue, we propose an a-priori model order reduction scheme based on the ECSW framework which does not require full order dynamic simulations to set up the ROM. A-priori schemes have been presented before for nonlinear (structural) dynamics. Multiple methods have been developed in the past to extend linear ROB approaches to nonlinear applications, e.g. by adding modal derivatives ${ }^{2}$. However, these approaches are only applicable to mild nonlinearities in practice. The concept of the modal derivatives was also extended through the dual modes approaches, as presented in Kim et al. $\underline{\underline{8}}$ and Mignolet et al. $\stackrel{9}{ }$, which allows to handle larger nonlinearities. The quadratic manifold lifting approach by Jain $\&$ Tiso $\underline{10}$ also presents an a-priori model order reduction scheme based on the modal derivative concept. Here this concept is also exploited to perform the hyper-reduction based on exploiting the lifted linearized response to select training vectors for the internal forces. Rutzmoser et al. $\stackrel{11}{\underline{1}}$ presented a novel approach based on Krylov theory to set up an a-priori reduced order basis and perform the element selection in an ECSW framework. Rutzmoser et al. $\stackrel{\underline{11}}{\underline{m}}$ also demonstrate the issue with employing training (simulation) based on modal-derivative based ROBs.

In this work we propose a pragmatic reduced order model setup for nonlinear structural models, based on the solution of a set of static problems and an $L_{1}$ optimal element selection. As the nonlinearity in many structural problems is strongly driven through geometric effects, and it are phenomena like element locking for large deformations which often cause large reduction errors, the proposed approach samples from a low number of largely deformed static solutions of the model to set up the ROB. In order to approximate the model dynamics, a modal basis is set up for these static reference configurations, which is similar to the dual modes approach ${ }^{8.9}$. Both the static deformation shapes and dynamic eigenmodes for the reference configurations are concatenated to set up the ROB, as discussed in Sec. 3. Due to this modal expansion around multiple static configuration, the proposed approach is denoted as the Multi-Expansion Modal (MEM) method. Starting from this ROB, a second aim is to obtain a low cost element selection procedure. In this work we propose to formulate the element selection as a non-negative linear programming problem $\frac{12}{2}$. It is known that these programs tend to stimulate sparse solutions, which is desirable to obtain a low cost

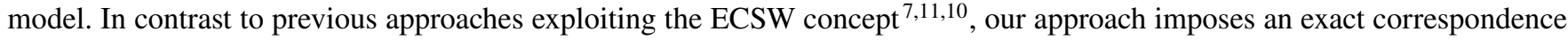
of some reduced internal force quantity in the constraints, such that only a single $L_{1}$ optimization needs to be performed. Even though these problems are $N P$-hard, many efficient approaches for (approximately) solving these $L_{1}$ optimization problems have been presented in literature and implemented in (open-source) software $\stackrel{13}{ }$. For the equality constraint two different choices are proposed: an equality constraint on a single reduced stiffness matrix or an equality constraint on the reference internal forces, as discussed in Sec.4. The different steps to consider when setting up a MEM model are then summarized in Sec.5. The proposed approach validated on a nonlinear cantilever beam model in Sec.6 and are shown to provide compact models at a low setup and simulation cost. 


\section{2 | PROBLEM STATEMENT: NONLINEAR ELASTODYNAMIC FINITE-ELEMENT MODEL}

The models considered in this work are obtained from a finite-element spatial semi-discretization of nonlinear elastic and elastodynamic systems. The proposed method is applicable to both static and dynamic models, but in this work the focus is on dynamic applications. The full order models (FOM) finite element models considered in this work, are described by an ordinary differential equation as:

$$
\mathbf{M} \ddot{\mathbf{x}}+\mathbf{f}^{i n t}(\mathbf{x})=\mathbf{f}^{\text {ext }},
$$

with the DOF vector $\mathbf{x} \in \mathbb{R}^{n}$, a constant mass matrix $\mathbf{M} \in \mathbb{R}^{n \times n}$, (nonlinear) internal force vector $\mathbf{f}^{\text {int }} \in \mathbb{R}^{n}$ and external force vector $\mathbf{f}^{\text {ext }} \in \mathbb{R}^{n}$. For nonlinear structural models expressed semi-discretized through a finite-element approach, the internal forces are obtained from the contributions of the elements:

$$
\mathbf{f}^{i n t} \mathbf{x}=\sum_{i=1}^{n_{e}} \mathbf{S}_{e, i} \mathbf{f}_{e, i}^{\text {int }}\left(\mathbf{x}_{e, i}\right)
$$

where $\mathbf{f}_{e, i}^{i n t} \in \mathbb{R}^{n_{e, i}}$ are the internal force contributions for element $i$ (with $n_{e, i}$ DOFs) and $\mathbf{S}_{e, i} \in \mathbb{R}^{n \times n_{e, i}}$ is a selection matrix which distributes these element contributions to the corresponding DOFs.

For realistic problems, these model typically consist of a large number of DOFs $n$ and many nonlinear function evaluations in $\mathbf{f}^{\text {int }}$. The number of DOFs and elements is easily in excess of ten-thousands. This leads to large computational loads both due to the systems inversion required for the time-integration, due to the large number of DOFs, and due to the model evaluation, due to the large number of elements. In dynamic applications, this issue is moreover compounded by the large number of timesteps for which the model typically needs to be solved.

In order to alleviate this computational burden, model order reduction can be employed. The overall nonlinear model order reduction scheme proposed by many authors $\frac{3,14.4}{}$ (and in this work) consists of two main steps:

- Set up a reduced order basis (ROB) $\mathbf{V}$ to approximate the full order model DOFs $\mathbf{x}$ in a low order space:

$$
\mathbf{x} \approx \mathbf{x}^{0}+\mathbf{V q},
$$

with a constant reduced order basis $\mathbf{V} \in \mathbb{R}^{n \times m}$ and a reference configuration $\mathbf{x}^{0} \in \mathbb{R}^{n}$. This expresses the relation between the FOM DOFs $\mathbf{x} \in \mathbb{R}^{n}$ and ROM DOFs $\mathbf{q} \in \mathbb{R}^{m}$.

- Set up an approximation of the nonlinear internal forces $\mathbf{f}^{\text {int }}$ after Galerkin projection in order to reduce the evaluation cost of the equations of motion:

$$
\overline{\mathbf{f}}^{i n t}(\mathbf{q}) \approx \tilde{\mathbf{f}}^{i n t}(\mathbf{q})=\mathbf{V}^{T} \mathbf{f}^{i n t}\left(\mathbf{x}^{0}+\mathbf{V q}\right)
$$

where $\tilde{\mathbf{f}}^{i n t}(\mathbf{q}) \in \mathbb{R}^{m}$ is the Galerkin projected internal force and $\overline{\mathbf{f}}^{\text {int }} \in \mathbb{R}^{m}$ is an approximation of this force which can be evaluated at a lower cost. For general nonlinear models, this approximation is typically performed based on the selection of dominant terms in the nonlinear equations and through weighting/interpolating them ${ }^{53,14,4}$.

Through this scheme, the reduced equations of motion can be summarized as:

$$
\tilde{\mathbf{M}} \ddot{\mathbf{q}}+\overline{\mathbf{f}}^{i n t}(\mathbf{q})=\tilde{\mathbf{f}}^{e x t},
$$

with the reduced mass matrix $\tilde{\mathbf{M}}=\mathbf{V}^{T} \mathbf{M V}, \in \mathbb{R}^{m \times m}$, the hyper-reduced (nonlinear) internal forces $\overline{\mathbf{f}}^{\text {int }} \in \mathbb{R}^{m}$ and the projected external forces $\tilde{\mathbf{f}} \tilde{\mathbf{e}}^{\text {ext }}=\mathbf{V}^{T} \mathbf{f}^{\text {ext }}, \in \mathbb{R}^{m}$.

The issue with most existing approaches following this scheme, is that they rely on performing full order training simulations in order to identify the ROB and most dominant nonlinear terms. In many practical applications however, it is infeasible to perform the full order simulations required for this training. In order to circumvent this issue, we propose a model order reduction scheme in this work which only requires a small set of static solutions for elastodynamic problems. This allows to set up a ROB and element selection which can be exploited in (long-term) dynamic simulations. As will be discussed in the following section, this information is exploited both for setting up the ROB and internal force approximation.

\section{3 | REDUCED ORDER BASIS SELECTION: MULTI-EXPANSION MODAL REDUCTION}

In this work we propose the multi-expansion modal (MEM) approach to provide a practical, if not mathematically rigorous, approach for obtaining a reduced order basis to perform dynamic simulations of (geometrically) nonlinear structural finite element models. This approach is an extension of the typical modal reduction approaches employed in small deformation structural 

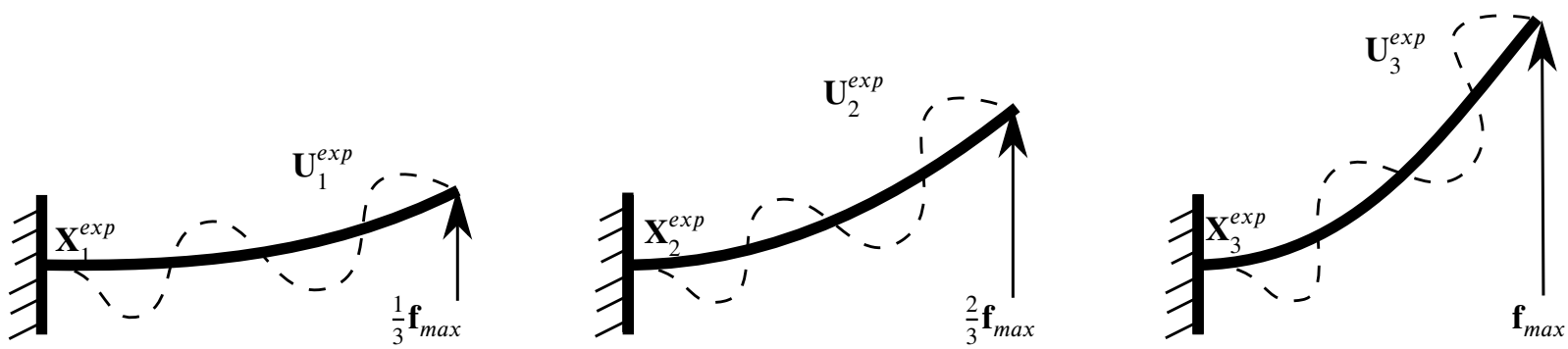

FIGURE 1 Multi-expansion point modal reduction construction

dynamics 1 . However, as is generally known, a purely modal decomposition is not suitable for nonlinear problems as they typically cause locking phenomena ${ }^{2}$. To circumvent this issue, the use of modal derivatives has been proposed ${ }^{2,11}$. However, from the authors' experience this approach is not robust for different nonlinear FE descriptions and might only marginally increase the non-locking region.

The assumption at the basis of the MEM approach is that the largest deformations are key in describing the nonlinear behavior of the system and should therefore be captured accurately. From an a-priori perspective, the largest deformations are assumed to be driven by the expected loads during the dynamic simulation which cause the largest linearized deformation. A common approach to compute the static response of a nonlinear FE model for a large external load, is to step-wise $\left(n_{\text {exp }}\right.$ intermediate steps) apply this load to obtain better convergence of e.g. a Newton-Raphson solver. This process is shown in Fig. 1 This leads

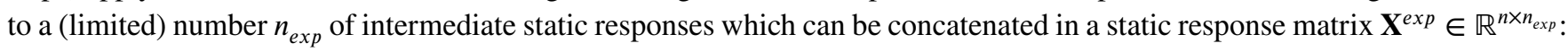

$$
\mathbf{X}^{\text {exp }}=\left[\mathbf{X}_{1}^{\exp }, \ldots, \mathbf{X}_{n_{\text {exp }}}^{\text {exp }}\right] .
$$

Each of these intermediate static responses $\mathbf{X}_{i}^{\text {exp }}$ again serves as an expansion point around which a modal basis $\mathbf{U}_{i}^{\text {exp }} \in \mathbb{R}^{n \times n_{m}}$ is computed (with $n_{m}$ the number of eigenmodes computed for each intermediate step)1:

$$
\mathbf{U}_{i}^{\text {exp }}=\operatorname{eig}\left(\mathbf{K}\left(\mathbf{X}_{i}^{\text {exp }}\right), \mathbf{M}\right)
$$

where $\mathbf{K} \in \mathbb{R}^{n \times n}$ is the stiffness matrix for the current static configuration $i$ :

$$
\mathbf{K}\left(\mathbf{X}_{i}^{e x p}\right)=\left.\frac{\partial \mathbf{f}^{i n t}}{\partial \mathbf{x}}\right|_{\mathbf{X}_{i}^{\exp }} .
$$

The resulting eigenbases $\mathbf{U}_{i}^{\text {exp }}$ are concatenated together in $\mathbf{U}^{\exp } \in \mathbb{R}^{n \times\left(n_{\text {exp }} \cdot n_{m}\right)}$ :

$$
\mathbf{U}^{\text {exp }}=\left[\mathbf{U}_{1}^{\exp }, \ldots, \mathbf{U}_{n_{\text {exp }}}^{\exp }\right] .
$$

We refer to this method as the multi-expansion point modal reduction (MEM) approach, as it considers a model decomposition around multiple expansion points. Conceptually this scheme can also be adopted for the exploitation of other linear model order reduction techniques like Krylov bases or balanced truncation 1 instead of the local modal decomposition, but in this work we focus on the modal approach which is somewhat of a standard in structural dynamics.

Starting from this collection of static deformations and dynamic modes, the reduced order basis is constructed. This construction is based on the following two steps:

- From the computed static configuration, a set of static deformation modes is computed:

$$
\Delta \mathbf{X}^{\exp }=\left[\mathbf{X}_{1}^{\exp }-\mathbf{x}^{0}, \ldots, \mathbf{X}_{n_{\text {exp }}}^{\text {exp }}-\mathbf{x}^{0}\right]
$$

A singular value decomposition 2 is performed on this static deformation matrix to retreive the $m_{s}$ dominant contributions $\mathbf{V}^{s} \in \mathbb{R}^{n \times m_{s}}$ :

$$
\begin{aligned}
\mathbf{U} \Xi \mathbf{W}^{T} & =\operatorname{svd}\left(\Delta \mathbf{X}^{\text {exp }}\right), \\
\mathbf{V}^{s} & =\left\{\mathbf{u}_{i}, i \in\left[1, \ldots, m_{s}\right]\right\} .
\end{aligned}
$$

\footnotetext{
${ }^{1}$ In this work we denote $\mathbf{U}=\operatorname{eig}(\mathbf{K}, \mathbf{M})$ as an algorithm to compute a second order eigenvalue decomposition $\mathbf{K U}=\mathbf{M U} \boldsymbol{\Lambda}$ with $\mathbf{\Lambda}$ a diagonal matrix containing the respective eigenvalues for the eigenmodes.

${ }^{2}$ In this work svd denotes a singular value decomposition algorithm
} 
For a limited number expansion points, one can simply set $m_{s}=n_{\text {exp }}$. This first part of the ROB should allow to cover large ranges of motion and to mitigate locking phenomena typically encountered in reduced geometrically nonlinear FE models.

- The full expanded modal basis $\mathbf{U}^{\exp }$ is orthogonalized with respect to $\mathbf{V}^{s}$ and mass-normalized, leading to $\mathbf{U}_{\perp}^{\exp }$. A second singular value decomposition is performed on this basis in order to obtain a constant approximation for the modal behavior of the nonlinear system by retaining the $m_{d}$ dominant contributions in $\mathbf{V}^{m} \in \mathbb{R}^{n \times m_{d}}$ :

$$
\begin{aligned}
\mathbf{U} \Xi \mathbf{W}^{T} & =\operatorname{svd}\left(\mathbf{U}_{\perp}^{\text {exp }}\right), \\
\mathbf{V}^{m} & =\left\{\mathbf{u}_{i}, i \in\left[1, \ldots, m_{d}\right]\right\} .
\end{aligned}
$$

Again, for a limited number of expansion points and a sufficiently small modal basis computed for each expansion point a choice of $m_{d}=n_{\text {exp }} \cdot n_{m}$ can be a feasible choice.

The final ROB $\mathbf{V} \in \mathbb{R}^{n \times m}$ (with $m=m_{s}+m_{d}$ ) is obtained by concatenating both the static deformation contributions and dynamic local modal contributions:

$$
\mathbf{V}=\left[\mathbf{V}^{s}, \mathbf{V}^{m}\right] .
$$

Even though the proposed MEM approach requires the solution of number of nonlinear static responses, this can generally be limited to the computation of a low number of static responses (e.g. ten steps), which is much lower than the number of nonlinear function evaluations for any realistic dynamics simulation.

Contrary to a dynamic training, the different static deformations can also be computed in parallel to further lower the training cost. Moreover, as discussed in Sec. 5, the reduced order model approach can already be exploited in order to also reduce this initial static setup cost when computed serially.

As with any modal reduction technique it is not possible to provide rigorous proofs for the accuracy of the resulting ROM. However the numerical example presented in Sec.6 demonstrates that this is a valid approach.

\section{4 | HYPER-REDUCTION: $L_{1}$ OPTIMAL ELEMENT SELECTION FOR MEM}

In order to further limit the computational load from the model evaluation, a low-cost approximation has to be made to evaluate the projected nonlinear internal forces. This approximate reduction step is referred to as hyper-reduction.

The hyper-reduction scheme proposed in this work is based on the energy-conserving weighting and sampling (ECSW) pro-

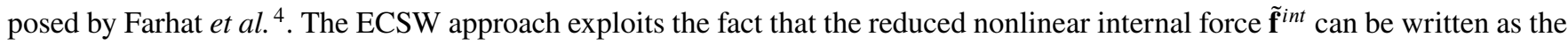
sum of the contributions from the different elements, respectively projected onto the ROB in a Galerkin framework:

$$
\tilde{\mathbf{f}}^{i n t}(\mathbf{q})=\mathbf{V}^{T} \mathbf{f}^{i n t}\left(\mathbf{x}^{0}+\mathbf{V q}\right)=\sum_{i=1}^{n_{e}} \mathbf{V}_{e, i}^{T} \mathbf{f}_{e, i}^{i n t}\left(\mathbf{x}_{e, i}\right),
$$

where $n_{e}$ is the number of elements in the model, $\mathbf{V}_{e, i} \in \mathbb{R}^{n \times n_{e, i}}$ and $\mathbf{x}_{e, i} \in \mathbb{R}^{n_{e, i}}$ are respectively the contributions of the ROB $\mathbf{V}$ and the full DOF vector at the nodes of element $i$, and $\mathbf{f}_{e, i}^{\text {int }}$ are the internal forces evaluated for element $i$. The ECSW framework proposes to approximate the internal forces as 4 :

$$
\tilde{\mathbf{f}}^{i n t}(\mathbf{x}) \approx \overline{\mathbf{f}}^{i n t}(\mathbf{x})=\sum_{i=1}^{n_{e}} \mathbf{V}_{e, i}^{T} \mathbf{f}_{e, i}^{i n t}\left(\mathbf{x}_{e, i}\right) \mathbf{s}_{i}, \quad s_{i} \geq 0
$$

where $\mathbf{s} \in \mathbb{R}_{+}^{n_{e}}$ is a non-negative weighting vector which should be chosen such that $\overline{\mathbf{f}}^{\text {int }}$ approximates $\tilde{\mathbf{f}}^{\text {int }}$ while setting as many elements as possible in $\mathbf{s}$ to zero. For all elements $i$ where $\mathbf{s}_{i}=0$ no internal force evaluation is required, an thus the computational load can be reduced. Moreover, by choosing $\mathbf{s} \leq 0$ the stability of the underlying FE model is preserved, which is essential for structural dynamics simulations ${ }^{4}$. In order to find $\mathbf{s}$ such that a maximal number of elements equals zero to obtain an approximation for the internal forces, a sparse optimization problem needs to be solved. This reduces the number of elements which need to be evaluated from $n_{e}$ to $n_{s}$. This sparse optimization problem, which is generally NP-hard, can be formulated such that it can be solved more efficiently in practice by exploiting a range of sparsity promoting optimization techniques ${ }^{\underline{7}}$. In the ECSW scheme, $\mathbf{s}$ is chosen to such that the approximate projected internal force best matches the projected internal forces obtained from a (or multiple) dynamic training simulation(s). This approach for the ROM setup is relatively expensive for two reasons: 
- Expensive training simulation have to be performed. For one-off simulations and many other cases, this initial computational load can be too high for practical exploitation of the MOR approach.

- Involved optimization schemes need to be set up in order to sequentially improve/degrade the accuracy of the approximate internal forces and/or sequentially add elements for which the weights are optimized ${ }^{7}$. As each separate optimization problem can get rather expensive in the case of many training scenarios and/or large models, this leads to a considerable overall computational load.

This approach is therefor not directly suitable for a-priori model reduction.

In this work we propose two alternative element sampling approaches which allow to exploit the information from the a-priori basis setup, as discussed in Sec. 3. The main motivation to choose for an element sampling approach, as proposed by Farhat $e t$ $a l . \stackrel{4}{ }$, is that this allows for an framework to automatically incorporate any type of nonlinearity described by the available original elements.

The aim of the two newly proposed approaches is to provide a more standardized optimization problem to allow for rapid element sampling and weighting when limited training data is available. These two approaches respectively select the elements based on:

- matching the projected stiffness matrix around the reference configuration (see Sec.4.1);

- matching (a subset of) the projected static internal forces for the expansion points (see Sec.4.2).

Rather than the tradeoff between sparseness of the element set and accuracy, the proposed approach employs an equality constraint to match the desired properties exactly. In general this would lead to obtaining the sparsest non-negative $\mathbf{s}$ is an $L_{0}$ optimization problem for these two cases:

$$
\begin{array}{ll}
\min _{\mathbf{s} \in \mathbb{R}_{e}} & |\mathbf{s}|_{0} \\
\text { subject to } & \mathbf{A s}=\mathbf{b}, \\
& \mathbf{s} \geq 0 .
\end{array}
$$

Here, the exact form of the equality constraint terms $\mathbf{A} \in \mathbb{R}^{n_{b} \times n_{e}}$ and $\mathbf{b} \in \mathbb{R}^{n_{b}}$ is dependent on which of the two approaches is selected. For a sufficient reduction in model size it is to be expected that $n_{b}<<n_{e}$, such that this equality constraint is underdetermined. However, as is generally known, this $L_{0}$ problem is NP-hard and computationally intractable for any realistic mesh size. In order to circumvent this issue, we propose to reformulate the problem as an $L_{1}$ optimization problem:

$$
\begin{array}{ll}
\min _{\mathbf{s} \in \mathbb{R}^{n_{e}}} & |\mathbf{s}|_{1} \\
\text { subject to } & \mathbf{A s}=\mathbf{b}, \\
& \mathbf{s} \geq 0 .
\end{array}
$$

In recent years, many authors have performed studies on the applicability of $L_{1}$ optimization in order to extract sparse solutions for underdetermined fitting problems and have shown this to be an effective approach ${ }^{12.7}$. An additional important benefit of the regular $L_{1}$ approach is the fact that many computational toolboxes exist to solve this optimization problem efficiently, which can be exploited to perform the element selection even on very large meshes. This is particularly appealing for those applications where very fine meshes are generated from auto-meshers, but only a (very) small number of motion modes are relevant for the model behavior, e.g. in the case of low-frequency excitation.

In the following two subsections (Sec.4.1 and 4.2), we discuss how this optimization problem is set up for the two proposed approaches.

\section{1 | Stiffness based element selection}

In the stiffness based element selection (SBS), we propose an element sampling approach based on a linearisation around a single reference configuration $\mathbf{x}^{r e f}$ for the nonlinear internal forces. The element sampling is set up to obtain an exact match for the hyper-reduced stiffness with the projected full stiffness matrix for this reference configuration:

$$
\tilde{\mathbf{K}}\left(\mathbf{x}^{r e f}\right)=\overline{\mathbf{K}}\left(\mathbf{x}^{r e f}\right)=\sum_{i=1}^{n_{e}} \mathbf{V}_{e, i}^{T} \mathbf{K}_{e, i}\left(\mathbf{x}_{e, i}^{r e f}\right) \mathbf{V}_{e, i} s_{i} .
$$


Again $\mathbf{s}$ should be selected to be non-negative and as sparse as possible. Given any reference configuration for the model and the ROB, this approach can be applied a-priori without training. Without any additional information, the reference configuration can be chosen as the undeformed configuration $\mathbf{x}^{0}$.

In this approach, the approximation of the projected stiffness for the reference configuration manifests itself as an equality constraint in Eq. (17).

In order to express the equality constraint for the reduced stiffness matrix, the matrices are vectorized:

$$
\overrightarrow{\mathbf{K}}=\left[\begin{array}{c}
\mathbf{K}_{11} \\
\cdots \\
\mathbf{K}_{n 1} \\
\cdots \\
\mathbf{K}_{1 n} \\
\cdots \\
\mathbf{K}_{n n}
\end{array}\right] .
$$

With this notation, the equality constraint equation can be written as:

$$
\left[\overrightarrow{\mathbf{V}_{e, 1}^{T} \mathbf{K}_{e, 1}\left(\mathbf{x}_{e, 1}^{r e f}\right) \mathbf{V}_{e, 1}}, \ldots, \overrightarrow{\mathbf{V}_{e, n_{e}}^{T} \mathbf{K}_{e, n_{e}}\left(\mathbf{x}_{e, n_{e}}^{r e f}\right) \mathbf{V}_{e, n_{e}}}\right] \mathbf{s}=\overline{\tilde{\mathbf{K}}\left(\mathbf{x}^{r e f}\right)},
$$

or in shorthand form as:

$$
\mathbf{A}^{K} \mathbf{s}=\overline{\tilde{\mathbf{K}}\left(\mathbf{x}^{r e f}\right)} .
$$

with $\mathbf{A}^{K} \in \mathbb{R}^{\left(m^{2}+m\right) / 2 \times n_{e}}$ after the symmetric elements in the stiffness matrix are eliminated. Several measures can be taken in order to improve the conditioning of this constraint optimization problem:

- As the (exact) stiffness matrix is always symmetric, the symmetric elements can be removed from equality constraint.

- The stiffness terms related to rigid body modes can also be removed, as these should also have zero-stiffness both for the assembled model and the elements separately.

Depending on the optimization toolbox used, it is useful to perform these eliminations prior to the optimization, or they will be easily handled by the optimizer.

This fitting problem always has a trivial solution for the constraint equations where $\mathbf{s}$ is a vector of ones, which is a nonnegative solution which matches the constraints. However, in most cases this will not be the sparsest solution. As is shown in the numerical validation in Sec. 6, regular optimization algorithms easily determine a s sparse non-trivial solution for this approach $\sqrt[3]{3}$ Due to the symmetry of the stiffness matrix, the optimization problem contains $\left(m^{2}+m\right) / 2$ constraint equations, and typically a solution is obtained with $\left(m^{2}+m\right) / 2$ nonzero elements in s. This trend will also be validated in Sec. 6. However, as this is a quadratic function of the reduced model size (which of course cannot exceed $n_{e}$ ), this also leads to a rapid rise in the number of elements and is therefore only desireable for sufficiently small reduced order models. In the case of larger reduced order models, the approach from Sec.4.2 might be preferable.

This approach also has some background from a physical perspective. As no additional approximation is performed on the mass matrix, besided the Galerkin projection, a matching of the stiffness matrix at the reference configuration ensures that the eigenfrequencies are preserved for small deformations with respect to this reference configuration. This is often desirable in elastodynamic applications. Moreover, as the selected elements should approximately correspond to those elements where the most energy is stored for a given set of nodes (assuming a consistent normalization of the ROB). These are therefor also the elements which can be expected to be most susceptible to nonlinear effects and the most important ones to include in the element selection to capture the nonlinear model behavior.

\section{2 | Reduced internal force based element selection}

A second approach which is put forward in this work is to exploit the internal forces encountered during the static training for the ROB setup, discussed in Sec 3 . In the force based element selection (FBS) the constraint equations which are added aim to

\footnotetext{
${ }^{3}$ In this work we do not investigate the convexity of the sparse optimization problem of this form further as the conditions to demonstrate convexity are difficult to show in practice and will most likely not apply to general nonlinear FE problems. Future work will focus on further formalizing when a well posed problem is expected.
} 
match the hyper-reduced and projected nonlinear internal forces for these static configurations $\mathbf{X}_{j}^{\exp }$ :

$$
\tilde{\mathbf{f}}^{i n t}\left(\mathbf{X}_{j}^{\text {exp }}\right)=\overline{\mathbf{f}}^{\text {int }}\left(\mathbf{X}_{j}^{\text {exp }}\right)=\sum_{i=1}^{n_{e}} \mathbf{V}_{e, i}^{T} \mathbf{f}_{e, i}^{\text {int }}\left(\left(\mathbf{X}_{j}^{\text {exp }}\right)_{e, i}\right) s_{i}, \quad \forall j \in\left[1, \ldots, n_{\text {exp }}\right] .
$$

Here again an $\mathbf{s}$ can be optimized for in order to minimize the number of elements which an non-zero contribution. In this case a set of representative internal forces can be recovered from the ROB setup. We assume that one exploits all available data, but a selection of a smaller subset could be used in order to further lower the computational load. With this full selection a total of $m \times n_{\text {exp }}$ constraint equations are present.

For every static configuration $j$, a set of $m$ constraint equations is added. These linear constraint equations can be obtained as:

$$
\left[\mathbf{V}_{e, 1}^{T} \mathbf{f}_{e, 1}^{\text {int }}\left(\left(\mathbf{X}_{j}^{\text {exp }}\right)_{e, 1}\right), \ldots, \mathbf{V}_{e, n_{e}}^{T} \mathbf{f}_{e, n_{e}}^{\text {int }}\left(\left(\mathbf{X}_{j}^{\text {exp }}\right)_{e, n_{e}}\right)\right] \mathbf{s}=\tilde{\mathbf{f}}^{\text {int }}\left(\mathbf{X}_{j}^{\exp }\right)
$$

or in shorthand form:

$$
\mathbf{A}^{f, j} \mathbf{S}=\tilde{\mathbf{f}}^{i n t}\left(\mathbf{X}_{j}^{\exp }\right)
$$

with $\mathbf{A}^{f, j} \in \mathbb{R}^{m \times n_{e}}$. For the full constraint set these are combined as:

$$
\mathbf{A}^{f} \mathbf{s}=\mathbf{b}^{f},
$$

with

$$
\begin{aligned}
\mathbf{A}^{f} & =\left[\begin{array}{c}
\mathbf{A}^{f, 1} \\
\vdots \\
\mathbf{A}^{f, n_{\text {exp }}}
\end{array}\right], \\
\mathbf{b}^{f} & =\left[\begin{array}{c}
\tilde{\mathbf{f}}^{i n t}\left(\mathbf{X}_{1}^{\exp }\right) \\
\vdots \\
\tilde{\mathbf{f}}^{i n t}\left(\mathbf{X}_{n_{\text {exp }}}^{\exp }\right)
\end{array}\right] .
\end{aligned}
$$

In the setup of these constraint equations, there is typically no structure present which can be exploited to simplify the problem. Care should only be taken that the static configurations used do not include the undeformed configuration, as this would lead to a trivial set of zero constraints. A trivial solution is again the case where $\mathbf{s}$ is a full vector of ones. However, the $L_{1}$ optimization properties should promote a sparse solution, in which case only $m \times n_{\text {exp }}$ nonzero elements should be present, as demonstrated in Sec.6. This is also a major advantage over the stiffness based approach from Sec.4.1, as now the number of elements only scales linearly with the ROM size. This approach assumes that only a limited number of reference static configurations are required in order to obtain a good approximation. However, as mentioned before, the number of elements selected can be further reduced by selecting only a subset of the intermediate static configurations.

\section{5 | MULTI-EXPANSION MODAL REDUCTION SETUP}

In Sec. 3 and Sec. 4 we presented concepts for both setting up a reduced order basis and element selection and weighting for the semi-a-priori model order reduction of elastodynamic models. In this section we summarize how a practical ROM setup can be performed following the MEM approach. A practical workflow (can) consists of following stages:

1. Select dominant excitation: A first choice to be made is the dominant excitation $\mathbf{f}^{\max }$ which determines the different expansion points. In many structural applications, the maximal load of the to-be simulated can be selected. In the case of multiple loading directions and anisotropic stiffness properties, a linearized model can be set up around the initial reference configuration to obtain an initial assessment of which load case will yield the maximum deformation and should be used for the static training. Overall this step will typically involve some domain specific knowledge to choose the most suitable reference excitation.

2. Select number of expansion points: After the selection of the reference static excitation $\mathbf{f}^{\text {max }}$, the number of expansion points $n_{\text {exp }}$ needs to be selected. For practical purposes the expansion loads $\mathbf{f}_{i}^{\text {exp }}$ are chosen equidistantly between the zero and maximum load:

$$
\mathbf{f}_{i}^{e x p}=\frac{i-1}{n_{\exp }-1} \mathbf{f}^{\max }
$$


where the first expansion point $i=1$ is always the undeformed configuration.

3. Select number of dynamic modes: Next the number of local dynamic modes $n_{m}$ and retained dynamic modes $m_{d}$ can be selected. This is a classical issue in structural dynamics modeling and model reduction, as exact error estimators for modal reduction techniques are not available. As a heuristic, the simulation engineer determines the frequency range of interest and selects the number of modes such that all modes within this frequency range are present. For convenience this can be evaluated on the undeformed reference configuration. As a practical rule of thumb, the authors propose to select $n_{m}=m_{d}$.

4. Select element selection approach: Finally the hyper reduction approach needs to be selected. In the case of a very low rank ROB, one can choose the stiffness based element selection as presented in Sec.4.1. In the case of a higher rank ROB, the stiffness based approach leads to a too extensive elements selection, and the force-based selection from Sec. 4.2 is preferable. From the authors' experience, and as demonstrated in the numerical validation in Sec.6 the element selection approach has only a secondary impact on the final ROM accuracy in comparison to the impact of the ROB.

5. Perform MEM ROM setup: During the MEM setup, the static deformations associated with the expansion points are evaluated consecutively. After the evaluation of expansion configuration $\mathbf{X}_{i}^{\text {exp }}$, the model is linearized in order to compute the $m_{d}$ eigenmodes $\mathbf{U}_{i}^{\text {exp }}$. Two options exist now:

- This process is repeated for all expansion points, and then all data is combined in order to set up the MEM model by computing the resulting ROB and performing the element selection.

- In order to reduce the cost of evaluating the expansion points, an intermediate MEM model can each time be constructed from the previously available data. This intermediate MEM model is then exploited to evaluate the new expansion point and the result from the MEM model is used as an initial guess for the iterator on the FOM at the new expansion point. Depending on the model size, MEM size and step size between two expansion points this can yield a considerable MEM setup cost reduction.

6. MEM ROM validation: As a first validation of a sufficient number expansion points and dynamic modes, a load bisecting two of the expansion points $\mathbf{f}^{b i s}=0.5\left(\mathbf{f}_{i}^{\text {exp }}+\mathbf{f}_{i+1}^{\text {exp }}\right)$ can be selected and the approximation error using the static expansion basis can be evaluated:

$$
\begin{aligned}
\mathbf{q}^{b i s} & \mapsto\left\{\left(\mathbf{V}^{s}\right)^{T} \mathbf{f}^{i n t}\left(\mathbf{V}^{s} \mathbf{q}^{b i s}\right)=\left(\mathbf{V}^{s}\right)^{T} \mathbf{f}^{b i s}, \quad \mathbf{q}^{b i s} \in \mathbb{R}^{n_{\text {exp }}}\right\}, \\
\epsilon & =\left|\mathbf{f}^{\text {int }}\left(\mathbf{V}^{s} \mathbf{q}^{\text {bis }}\right)-\mathbf{f}^{b i s}\right|_{2}
\end{aligned}
$$

if this error $\epsilon$ is too large, more expansion points need to be selected. However, the acceptable error on this approximation error can be highly domain dependent and difficult to assess in general. Also the dynamic character can be heuristically evaluated by considering the modal decomposition in the frequency range of interest for this bisecting configuration. If the error for the eigenfrequencies and/or eigenmodes exceeds a certain threshold, the number of expansion points can be further increased.

This reduction scheme with exploitation of the ROM for reducing the number FOM iterations, is summarized in Tab.

The development of a more automated MEM model setup is subject of future research.

\section{6 | NUMERICAL VALIDATION}

This section presents a numerical validation for the proposed MEM model order reduction methodology. In Sec.6.1 we present the validation model used in this work. In Sec.6.3 the choice of reduced order basis and hyper-reduction, as presented in Sec. 3 and Sec. 4 are validated. The obtained results are also compared to a model derivative approach without hyper-reduction for reference.

\section{1 | Model description}

The proposed methodology is validated on a geometrical nonlinear cantilever beam model. The geometric nonlinearity is modeled using corotational shell elements $\frac{15}{}$. This corotational formulation introduces higher order nonlinearities in the model, 
TABLE 1 Multi-Expansion Model reduced order model setup.

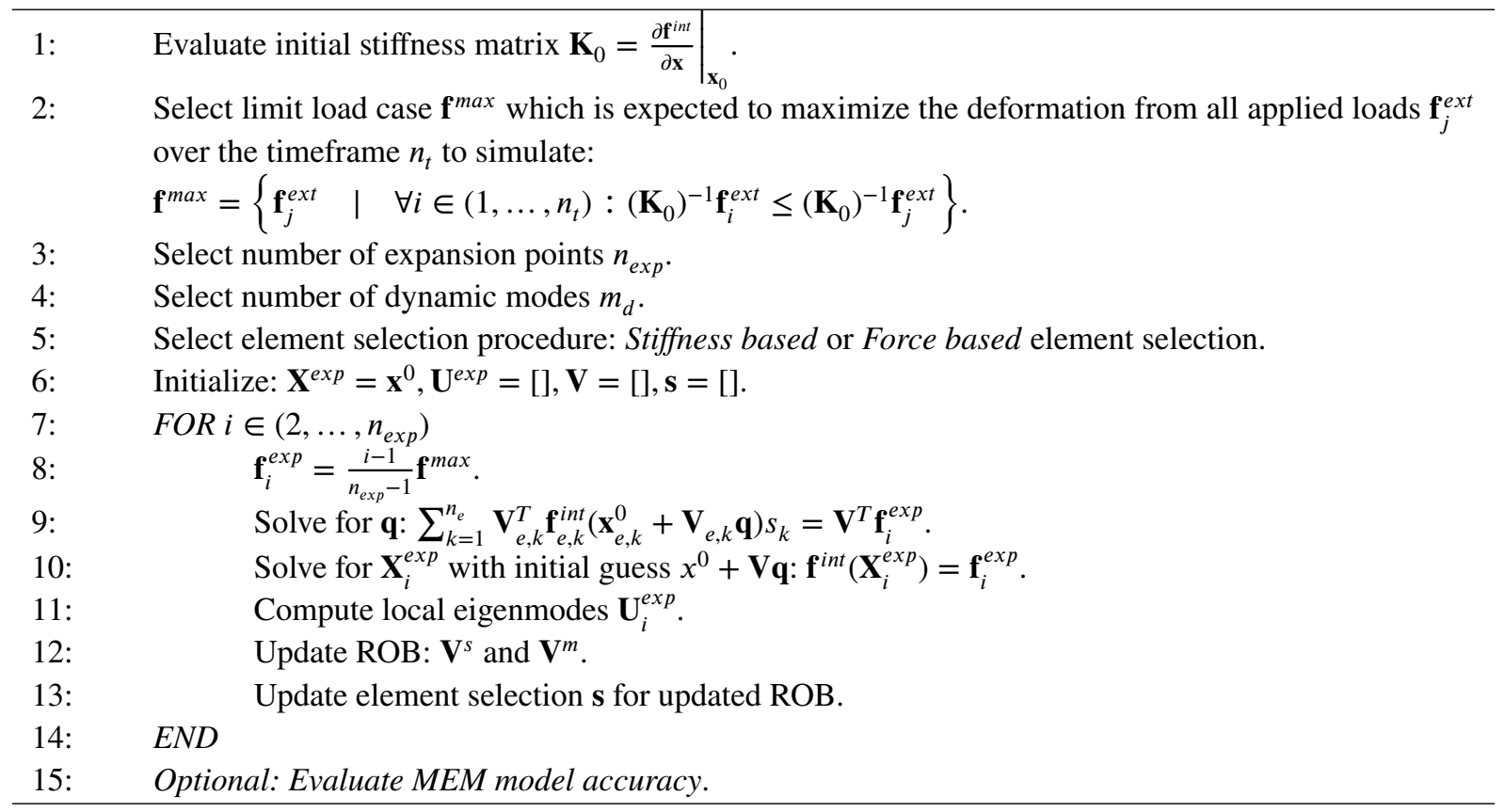

which are difficult to approximate using e.g. modal derivatives. This in turn makes is more challenging to prevent locking. The (deformed) model mesh is shown in Fig. 2 This model consists of 150 elements with a total of 1116 translational and rota-

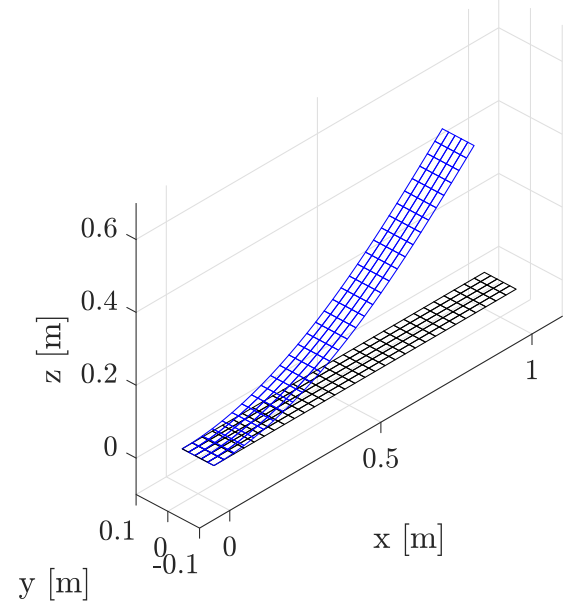

FIGURE 2 Cantilever beam with corotational shell elements and vertical tip load.

tional DOFs. The nonlinear finite element model and reduced order model are implemented in an in-house developed Matlab toolbox for corotational finite elements, where the ROM reuses the element implementation from the FOM. The lumped mass matrix and local linear element stiffness matrices are extracted from NX Nastran for CQUAD elements 16 and exploited in a corotational frame. The properties for the elements are shown in Table 2

For this validation, The applied tip load is distributed equally over the tip nodes. The course over time is shown in Fig. 3 The time integration is performed using a generalized alpha integrator with a timestep of $\Delta t=10^{-3} \mathrm{~s}$ and the numerical damping set to $\rho=0.7 \underline{17}$. 
TABLE 2 Cantilever beam element properties

\begin{tabular}{|c|c|}
\hline Thickness & $10 \mathrm{~mm}$ \\
Density & $7.8 \cdot 10^{3} \mathrm{~kg} / \mathrm{m}^{3}$ \\
Young's modulus & $210 \cdot 10^{9} \mathrm{~kg} / \mathrm{m}^{3}$ \\
Poison's ratio & 0.3 \\
\hline
\end{tabular}

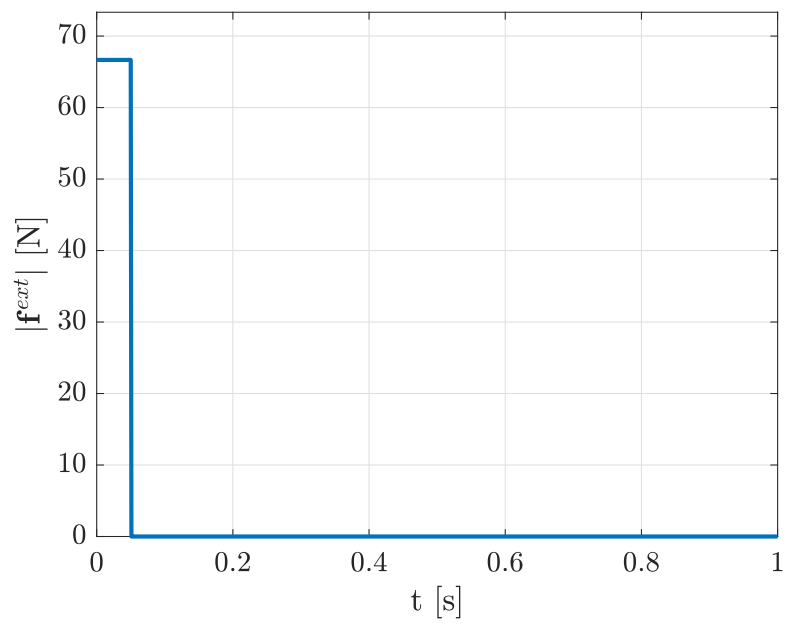

FIGURE 3 Tip force applied for dynamic cantilever beam simulation.

\section{2 | Simulation results}

As a simulation result, we first consider a static simulation to demonstrate the potential for locking in the presented model. For a model with $n_{\text {exp }}=4$ and $n_{m}=m_{d}=5$, the static vertical tip displacement and displacement error are shown in Fig. 4 for a purely projection based reduction. This figure compares the static response of a purely projected MEM model with the
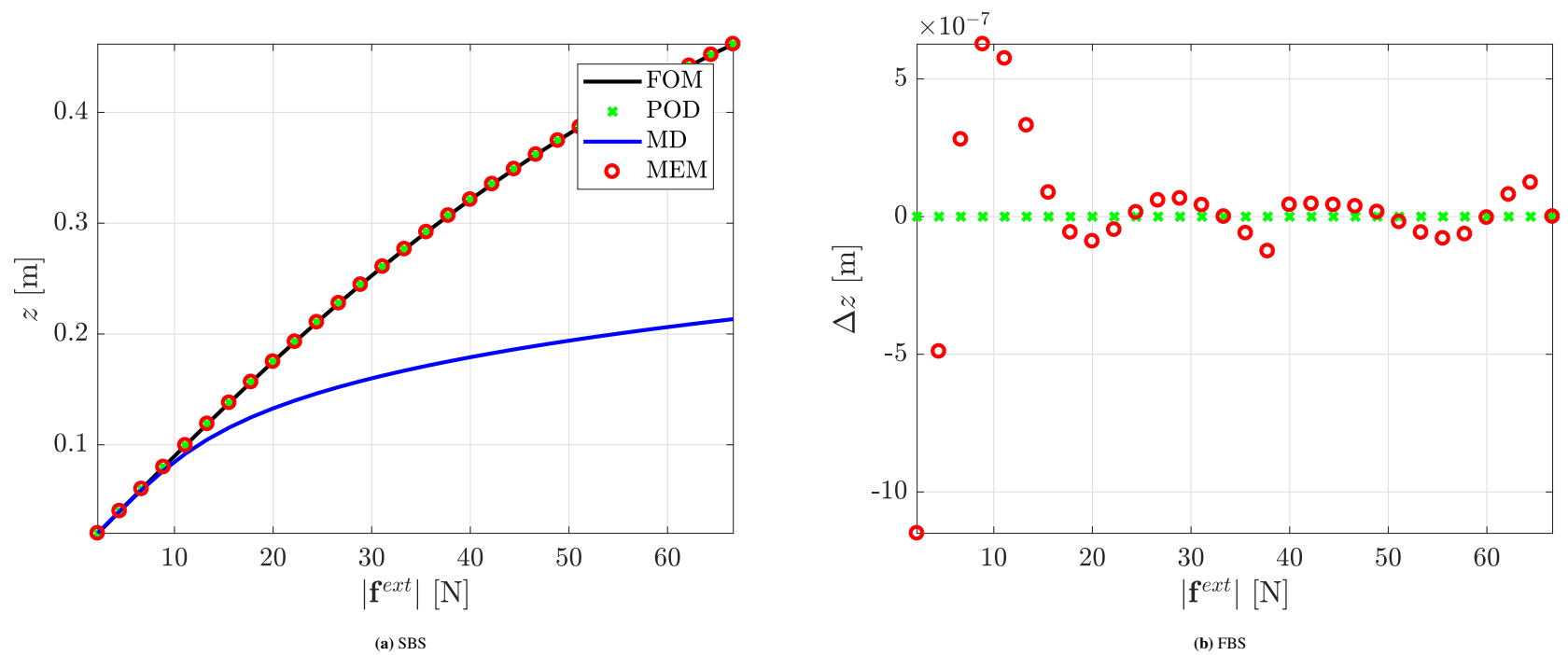

FIGURE 4 Static vertical tip displacement and error as a function of applied load for the FOM, a projected model with a POD ROB, a modal derivative (MD) ROB and the MEM ROB, all with $m=9$. 
response obtained from a POD model with the same size trained on the unreduced static response ( 30 samples) and with the response from a projected model from a modal derivative basis (MD). The modal derivative basis is obtained by accounting for the linear static and the first two dynamic eigenmodes, leading to a total $m=9$ by adding the respective modal derivatives. The modal derivative ROB is clearly inadequate to model the behavior with acceptable accuracy and leads to significant stiffening (locking) of the model. This approach is further omitted from the discussion. This figure also clearly shows the interpolatory behavior of the MEM approach between the expansion points, where the error is smaller at these expansion points. Here it is immediately clear that the modal derivative approach cannot accurately capture the nonlinear behavior and except at very small displacements, leads to relatively large errors due locking of the model.

The dynamic tip response for the cantilever beam (and error), comparing the results from the FOM, the MEM models $\left(n_{\text {exp }}=\right.$ 9, $n_{m}=m_{d}=3$ ), and a POD projected model $(m=10)$ is shown in Fig. 5 This figure clearly shows that both the MEM
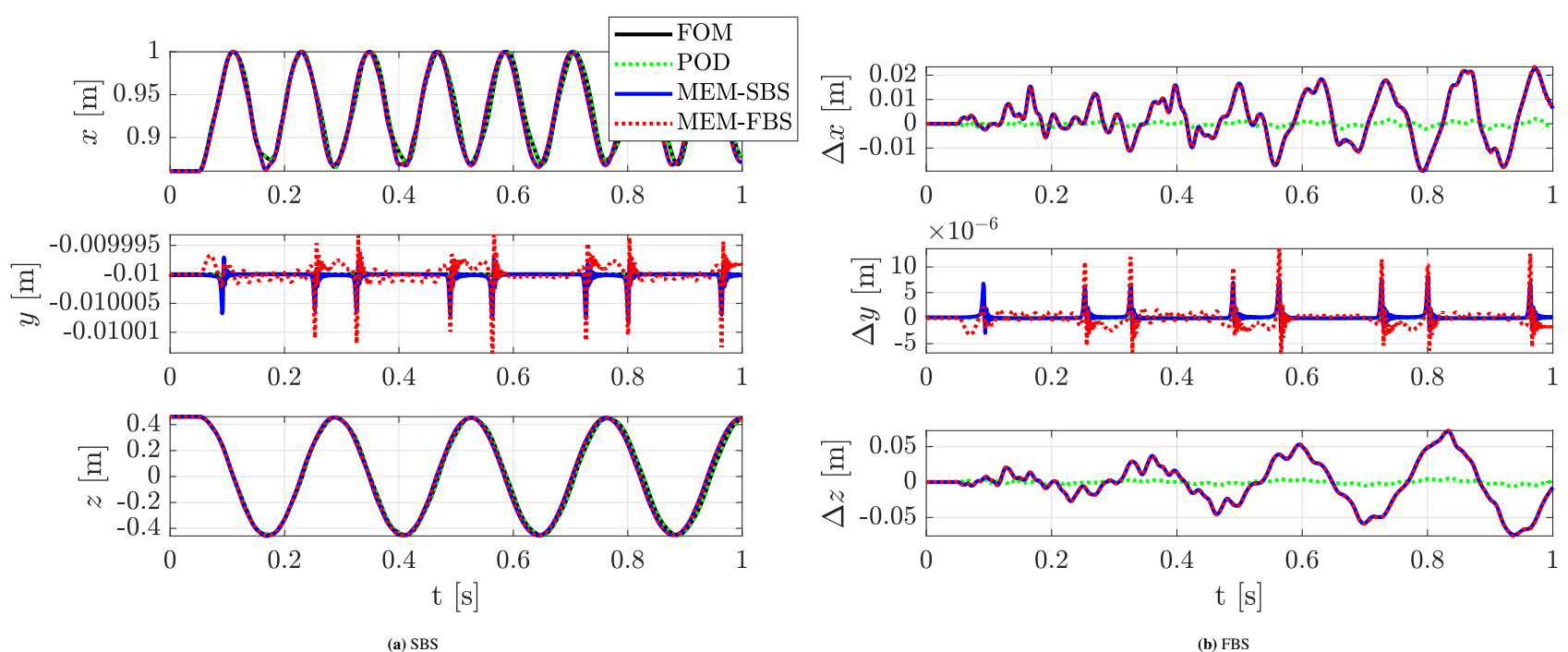

FIGURE 5 Comparison of dynamic tip response of cantilever beam for FOM, MEM model and POD projected model.

approach and the POD approach allow for accurate results, but the POD approach delivers results with very high accuracy as this is a fully reproductive simulation. Indicatively, the FOM reference simulation takes about $300 s$ on a laptop with an Intel Core i7-7700HQ CPU in the developed Matlab finite element toolbox. However the POD approach requires a full time-domain simulation to set up the model and hence increases the computational time for a single simulation when also considering the MOR setup time. The MEM models on the other hand takes $6 s$ to evaluate the seven expansion points and $1 s$ to perform the stiffness based and $0.1 s$ to perform the force based element selection respectively $y$. With both of the resulting MEM models the dynamic simulation only takes around 150 s, indicating an overall speed-up even for this limited size FOM.

\section{3 | MEM model convergence}

The convergence of the error for the dynamic simulation as a function of the MEM model size using both element selection approaches is shown in Fig. 6 For each curve, the number of expansion points $n_{\text {exp }}$ is kept constant and the number of dynamic modes $n_{m}=m_{d}$ is increased. As a reference this figure also shows the convergence for the use of a POD basis without hyperreduction. This figure clearly shows the importance of a sufficient number of expansion points in order to obtain an accurate response from the MEM approach. These figures moreover indicate that the choice of the element selection approach has a relatively negligible result on the MEM accuracy in comparison to the impact of the ROB size.

The number of elements selected by the two proposed approaches as a function of the ROB size are shown in Fig. 7 For performing the element selection in this work, the linprog-function from the MATLAB Optimization Toolbox is used with the

\footnotetext{
${ }^{4}$ The expansion point computation does not the exploit the reduced model in this implementation and the element selection is only performed once
} 

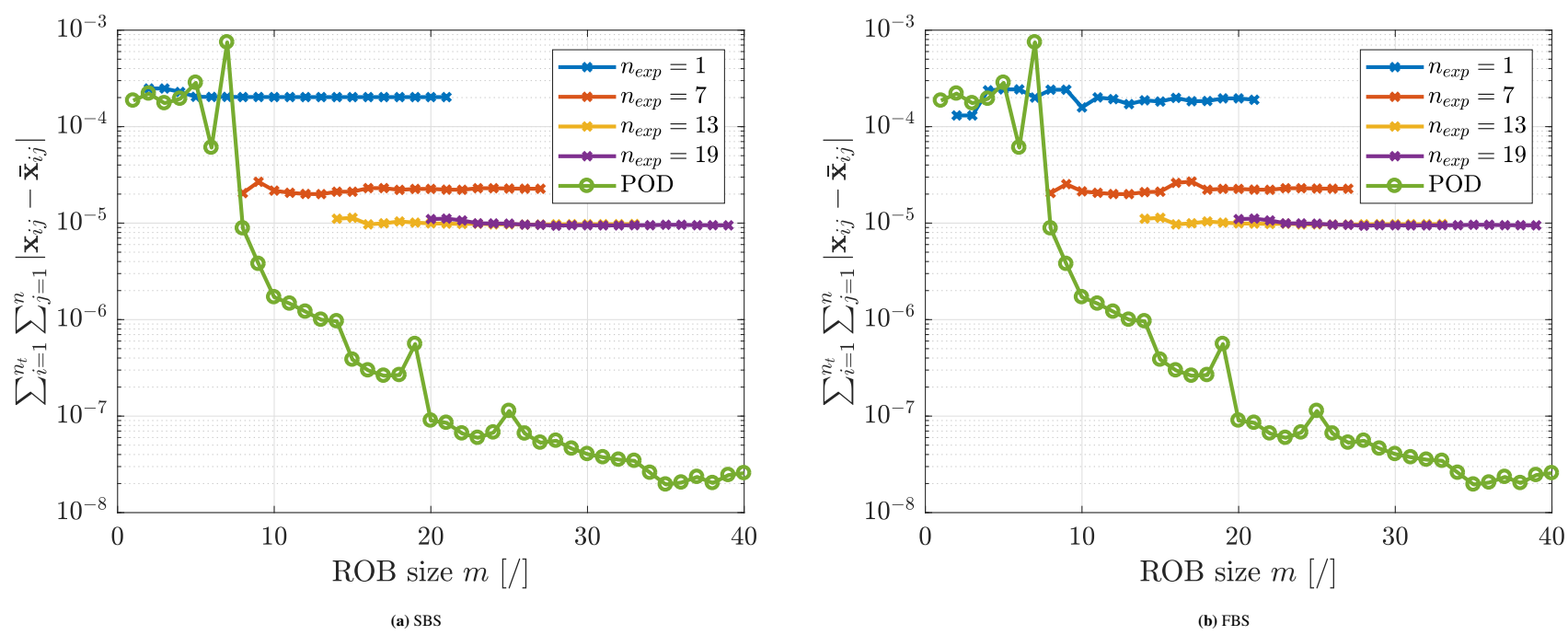

FIGURE 6 Convergence of simulation error for increasing ROB size

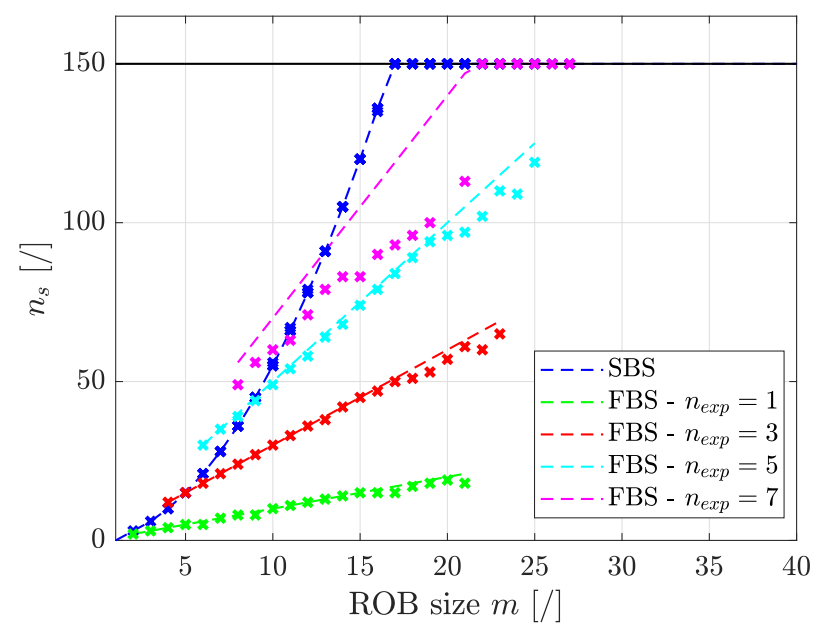

FIGURE 7 Number of selected elements for hyper-reduced model of cantilever beam (-- theoretical number of elements, $\times$ selected number of elements).

dual-simplex solver ${ }^{18}$. The default tolerances are used and in order to ensure proper sparsity of the final selection a treshold of $10^{-5}$ is taken into account on the obtained weights. Fig. 7 shows the increasing number of elements when the ROB size increases with the SBS and FBS. With the defaut optimizer settings, the number of selected elements is exactly the same or smaller than the theoretical number of elements which could be selected (as discussed in Sec. 4). This demonstrates the good sparse solution selection of the $L_{1}$ optimizer for the MEM hyper-reduction.

For reference Fig. 8 also shows which elements are selected using the stiffness and force based selection for the MEM model with $n_{\text {exp }}=7$ and $n_{m}=m_{d}=3(m=10)$. For this MEM size, the SBS approach selects 55 elements and the FBS approach selects 60 elements. It is interesting to notice that the SBS approach leads to a relatively uniform element selection whereas the FBS approach puts more emphasis on the (highly stressed) elements near the fixed boundary. For both approaches the elements near the center of the beam have higher weights associated with them. 


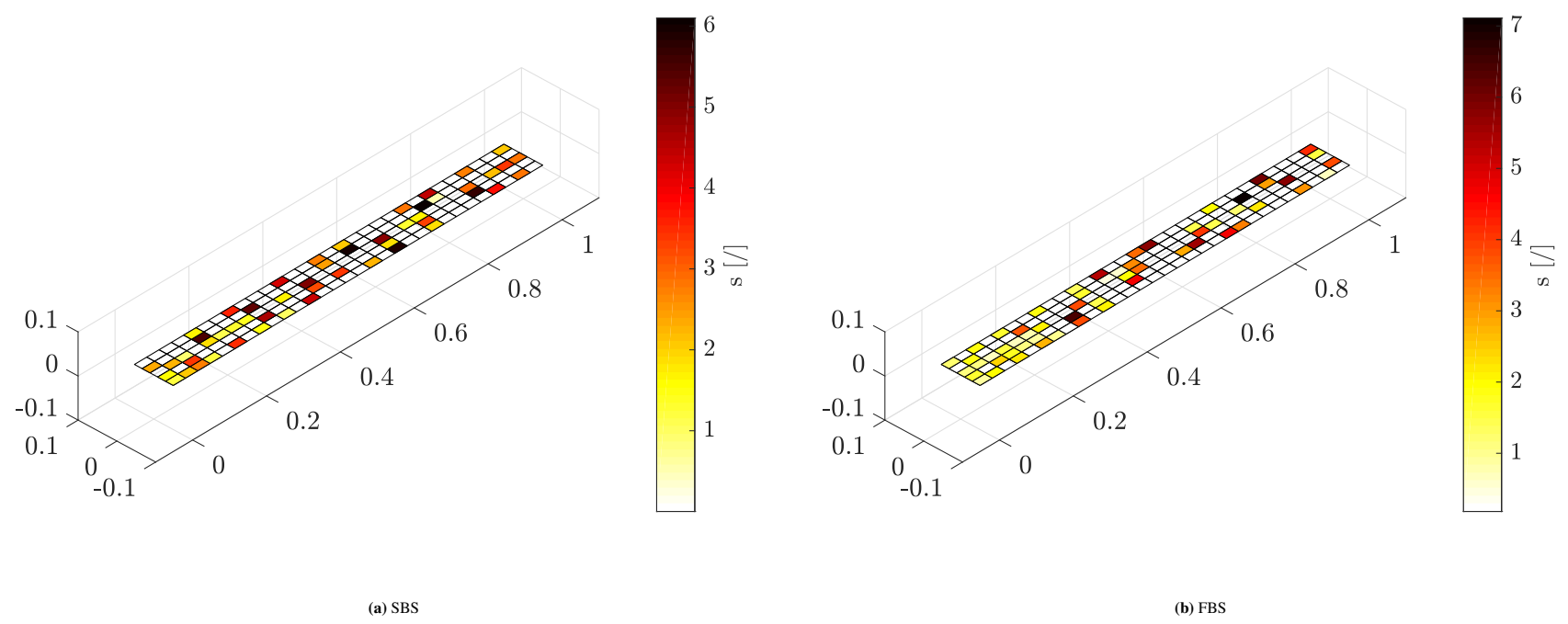

FIGURE 8 Selected elements with a MEM model size of $m=10$ for the stiffness based (left) and force based element selection (right) (element weight $s$ in color).

\section{7 | CONCLUSION}

In this work we propose a novel pragmatic framework which can be exploited for the reduced order modeling of nonlinear structural dynamics. The framework contains two key aspects: a reduced order basis (ROB) setup and a reduced element selection (hyper-reduction). Stemming from a long history in structural models, the ROB setup is based on a modal decomposition. However, in order to capture the main nonlinear effects, typically driven by large deformations in structural problems, multiple modal bases are computed for different deformed configurations and combined into a single ROB. The proposed method is therefore denoted as the Multi-Expansion Modal (MEM) approach. This provides an easy to interpret approach for structural engineers who classically have considerable experience in choosing modal bases for linear models. Starting from this ROB, an element selection is performed to lower the evaluation cost of the nonlinear model. The element selection is based on a non-negative $L_{1}$ optimization with a set of equality constraints, either for matching the reduced reference stiffness matrix, or for matching the static deformation forces. As the $L_{1}$ optimization promotes sparsity, typically a small number of elements is selected to accurately approach the reduced order model behavior. The performance of the proposed framework is validated on a numerical example of a geometrically nonlinear (corotational frame) cantilever beam model. The MEM approach is shown to have good convergence, with an easy to tune ROM. Depending on the ROB size, the two proposed element selection methods allow for a consistent number of selected elements, which makes it easy to a-priori tailor the model to the allowable computational cost. The exploitation of these concepts for an automated ROM setup is being investigated as future research, as well as the exploitation in nonlinear finite elements model for structural applications with a broad range of nonlinearities.

\section{ACKNOWLEDGEMENTS}

The research of Frank Naets is funded by a postdoctoral fellowship of the Fund for Scientific Research, Flanders (FWO). The Research Fund KU Leuven is gratefully acknowledged for its support.

\section{References}

1. Besselink B., Tabak U., Lutowska A., et al. A comparison of model reduction techniques from structural dynamics, numerical mathematics and systems and control. Journal of Sound and Vibration. 2013;332(19):4403-4422. 
2. Idelsohn Sergio R, Cardona Alberto. A reduction method for nonlinear structural dynamic analysis. Computer Methods in Applied Mechanics and Engineering. 1985;49(3):253-279.

3. Chaturantabut Saifon, Sorensen D.C.. Nonlinear model reduction via discrete empirical interpolation. SIAM Journal on Scientific Computing. 2010;32(5):2727-2764.

4. Farhat C., Avery P., Chapman T., Cortial J.. Dimensional reduction of nonlinear finite element dynamic models with finite rotations and energy-based mesh sampling and weighting for computational efficiency. International Journal for Numerical Methods in Engineering. 2014;98(9):625-662.

5. Barrault Maxime, Maday Yvon, Nguyen Ngoc Cuong, Patera Anthony T. An empirical interpolation method: application to efficient reduced-basis discretization of partial differential equations. Comptes Rendus Mathematique. 2004;339(9):667672.

6. Maday Yvon, Mula Olga, Patera AT, Yano Masayuki. The generalized Empirical Interpolation Method: stability theory on Hilbert spaces with an application to the Stokes equation. Computer Methods in Applied Mechanics and Engineering. 2015;287:310-334.

7. Chapman Todd, Avery Philip, Collins Pat, Farhat Charbel. Accelerated mesh sampling for the hyper reduction of nonlinear computational models. International Journal for Numerical Methods in Engineering. 2016;

8. Kim Kwangkeun, Radu Adrian G, Wang XQ, Mignolet Marc P. Nonlinear reduced order modeling of isotropic and functionally graded plates. International Journal of Non-Linear Mechanics. 2013;49:100-110.

9. Mignolet Marc P, Przekop Adam, Rizzi Stephen A, Spottswood S Michael. A review of indirect/non-intrusive reduced order modeling of nonlinear geometric structures. Journal of Sound and Vibration. 2013;332(10):2437-2460.

10. Jain S., Tiso P.. Simulation-free hyper-reduction for geometrically nonlinear structural dynamics: A quadratic manifold lifting approach. Journal of Computational and Nonlinear Dynamics. 2018;13(7).

11. Rutzmoser Johannes B, Rixen Daniel J, Tiso Paolo. Model order reduction using an adaptive basis for geometrically nonlinear structural dynamics. In: ; 2014.

12. Donoho David L, Tanner Jared. Sparse nonnegative solution of underdetermined linear equations by linear programming. Proceedings of the National Academy of Sciences of the United States of America. 2005;102(27):9446-9451.

13. Dantzig George B, Thapa Mukund N. Linear programming 1: introduction. Springer Science \& Business Media; 2006.

14. Tiso P., Rixen D.. Discrete Empirical Interpolation Method for Finite Element Structural Dynamics. In: ; 2013.

15. Felippa CA, Haugen B. A unified formulation of small-strain corotational finite elements: I. Theory. Computer Methods in Applied Mechanics and Engineering. 2005;194(21):2285-2335.

16. Software MSC. MSC.Nastran 2004 Quick Reference Guide http://www.mscsoftware.com/Products/CAE-Tools/MSCNastran.aspx; .

17. Brüls O., Arnold M.. The generalized-alpha scheme as a linear multistep integrator: Towards a general mechatronic simulator. ASME Journal of Computational and Nonlinear Dynamics. 2008;3(4).

18. MATLAB Optimization Toolbox. The MathWorks, Natick, MA, USA; 2017.

How to cite this article: Naets F., De Gregoriis, D., and Desmet, W. (2018), Multi-Expansion Modal Reduction: A pragmatic semi-a-priori model order reduction approach for nonlinear structural dynamics, Int. J. Num. Meth. Engng., 2018. 